TM-1504

\title{
Debunching Into a Bucket of Lower Harmonic Number
}

\author{
James A. MacLachlan and James E. Griffin \\ Fermi National Laboratory \\ P.O. Box 500, Batavia, Illinois 60510
}

December 9, 1987 


\title{
Debunching Into a Bucket of Lower Harmonic Number
}

\author{
James A. MacLachlan and James E. Griffin
}

9 December 1987

\begin{abstract}
The adiabatic debunching of beam from buckets of higher harmonic number into waiting buckets of lower harmonic number is a critical step in the current scheme of operation for Tev I. The optimum choice of $\mathrm{rf}$ system parameters for this "bunch coalescing" process is not immediately obvious. In this note two examples are presented along with generalizations based upon them and experience with the Tevatron I design which can simplify the selection of appropriate parameters for different conditions.
\end{abstract}

\section{Introduction}

The bunch coalescing process used in the Main Ring to change several ( 713) $h=1113$ bunches into a single bunch for colliding beams in the Tevatron (see Ref. [1]) depends on adiabatically debunching those bunches into an $h=53$ bucket in a manner nearly conserving longitudinal emmitence. A limitation in principle is the necessity to maintain the beam at very low momentum spread for a long time $(\sim 1 \mathrm{~s})$ to approximate an adiabatic process. Thus, microwave instability, by fixing a minimum stable momentum spread, will set a boundary for realistic parameters. Quite apart from practical considerations, however, it is not obvious how to choose the if system parameters to obtain the desired result even in the absence of instability. In the course of the Tevatron I design study a set of parameters for coalescing thirteen antiproton bunches was worked out and its efficacy in the absence of microwave instability was demonstrated in an ESME (Ref. [2]) simulation. Two somewhat different cases will be treated in this note to illustrate that there are some guidelines for a systematic selection of parameters. The antiproton bunch coalescing is qualitatively a time reversal of the $53 \mathrm{MHz}$ bunching process which makes the thirteen small bunches from one big one in the Accumulator. It is reasonable that, at least under ideal conditions, antiproton coalescing can be carried out with very little dilution. 


\section{Coalescing a Few Equal Bunches}

The example that is worked out here is somewhat like proton coalescing for the Tevatron in which the initial bunches have the same area. The particulars are chosen differently for didactic reasons. Because the bunch areas are not related in the manner resulting from a prior capture from a common bunch, one could anticipate some dilution in putting them into one bunch. It will be shown that on a practical scale the dilution can be insignificant.

Specifically, we calculate parameters for coalescing three $0.15 \mathrm{eVs}, \mathrm{h}=1113$, bunches in the Main Ring at $150 \mathrm{GeV}$ into a single $\mathrm{h}=159$ bunch. Table 1 gives the complete specification for this example. The three $h=1113$ buckets span \pm 77 deg of $h=159$ phase. The low-frequency voltage is chosen so that the contour in a bucket provided by the low-frequency voltage alone, containing the total emmitence of $0.45 \mathrm{eVs}$, will pass through the points $\varphi= \pm 77 \mathrm{deg}, \Delta E=$ 0 . The choice is plausible; experience suggests that it should be practically optimum. A lower voltage results in some of the debuching beam moving out too far in phase, including empty phasespace in the coalesced bunch as it streams around adjoining empty high-frequency buckets. Higher voltage results in the inclusion of empty phasespace as particles escaping from the outlying bunches pass outside the inner bucket before the $h=1113$ voltage has dropped enough to make it full. The debunching takes place by reducing the high-frequency voltage adiabatically in the presence of a fixed low-frequency voltage. The time scale for the voltage reduction is set by the synchrotron oscillation period in the low-frequency bucket because it is oscillation in this bucket that redistributes the charge. This characterization of the process is only heuristic because in fact the particles move on trajectories resulting from the simultaneous action of both rf systems. Even though independent $h=1113$ and $h=159$ buckets do not coexist, however, there is a set seven stable fixed points within $2 \pi$ of $h=159$ phase as long as there is $h=1113$ present. The simplified language is very convient for descriptive purposes.

In Fig. 1 are shown three $h=1113$ bunches of $0.15 \mathrm{eVs}$ in $0.27 \mathrm{eVs}$ buckets produced by $4 \mathrm{kV}$ of $53 \mathrm{MHz}$ rf. The $\mathrm{h}=159$ is present at $500 \mathrm{~V}$, a level at which the effect on the high-frequency buckets is negligible. The phasespace tracking program ESME is used to follow the evolution of this distribution as the $\mathrm{h}=1113 \mathrm{rf}$ is reduced iso-adiabatically over $2 \times 10^{5}$ turns. The small-amplitude synchrotron tune in a bucket produced by $500 \mathrm{~V}$ at $\mathrm{h}=159$ is $1.8 \times 10^{-5}$, so the reduction of the $h=1113$ voltage takes 3.6 periods of the $h=159$ oscillation. That the motion at the end of this time is reasonably pure $h=159$ is suggested by the bucket contour shown with the coalesced bunch in Fig. 2. The area of the resulting bunch from the scatter plot is $0.45 \mathrm{eVs}$ as closely as one can measure, viz., $\mathrm{O}(1 \%)$.

In the next three figures partially debunched results are shown for different levels of $h=159$. Figure 3 shows the distribution after $6 \times 10^{4}$ turns of the tracking which produced Fig. 2; the $500 \mathrm{~V}$ level of the low-frequency is nearly 


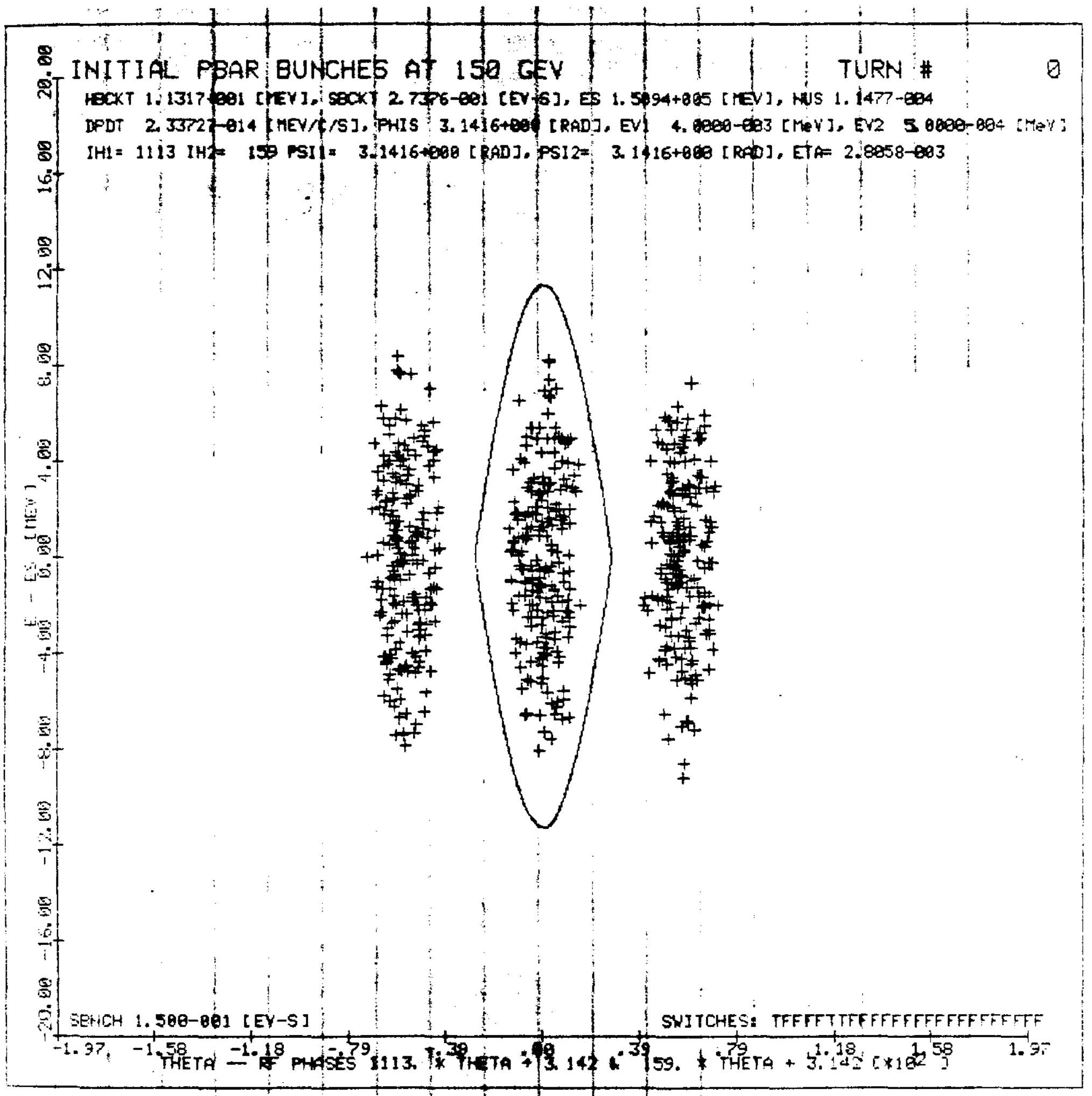

Figure 1: Initial $h=1113$ bunches and the central bucket contour 


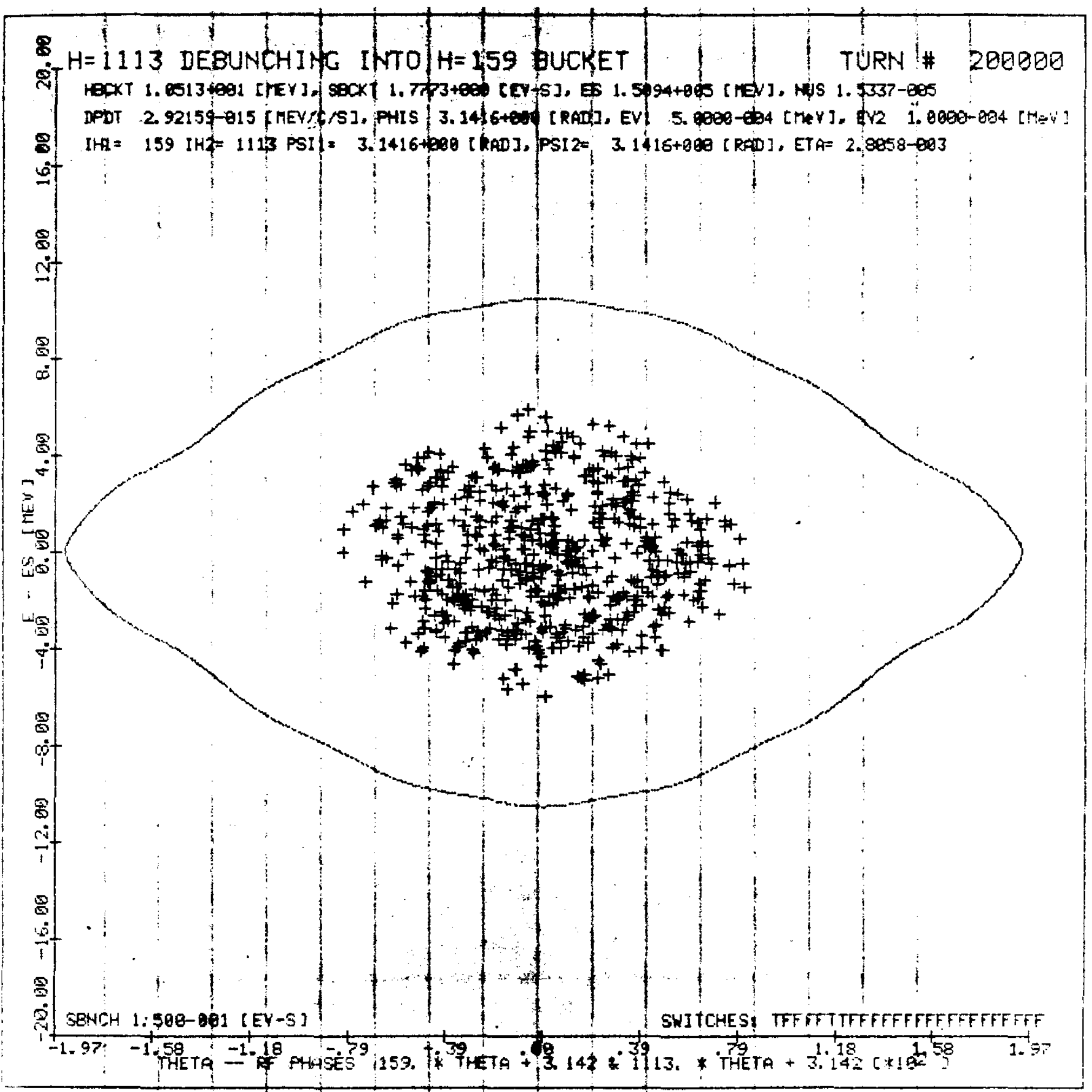

Figure 2: Coelesced $h=159$ bunch and two-frequency bucket contour 


\begin{tabular}{|c|c|c|}
\hline Orbit circumference $(2 \pi \bar{R})$ & 6.283 & km \\
\hline Transition gamma $\left(\gamma_{T}\right)$ & 18.75 & \\
\hline Harmonic number, 53 MHz system & 1113 & \\
\hline Initial $53 \mathrm{MHz}$ voltage $\left(V_{i}^{(1113)}\right)$ & 4.0 & \\
\hline Initial $h=1113$ bucket area & 0.27 & eVs \\
\hline Final $53 \mathrm{MHz}$ voltage $\left(V_{t}^{(1113)}\right)$ & 180. & V \\
\hline Final $h=1113$ bucket area & 0.06 & $\mathrm{eVs}$ \\
\hline Number of turns & $2 \times 10^{1}$ & \\
\hline Harmonic number, 7.5 $\mathrm{MHz}$ system & 159 & \\
\hline Voltage, $7.5 \mathrm{MHz}$ system $\left(V^{(15 \theta)}\right)$ & 500. & $\mathrm{~V}$ \\
\hline Bucket area for $h=159$ & 1.74 & eVs \\
\hline Number of $h=1113$ bunches & 3 & \\
\hline Initial $h=1113$ bunch areas & 0.15 & eVs \\
\hline Final $h=159$ bunch area & $\sim 0.45$ & eVs \\
\hline
\end{tabular}

Table 1: RF System Parameters for $h=1113$ to $h=159$ Coalescing

optimum. Note that the particles leaving the flanking bunches are drifting only toward the center bunch. As they pass around the separatrix for the center bunch there is no distinct void between the deconfined particles and those still confined to the central bucket. Figure 4 shows a corresponding result when the $\mathrm{h}=159$ voltage is set to $1420 \mathrm{~V}$. It shows that the particles deconfined from the flanking bunches start passing around the central bucket before it has shrunk enough to be full. The $176 \mathrm{~V}$ case shown in Fig. 5 demonstrates that the dilution occurs for too little $h=159$ voltage when deconfined particles are free to move out too far in phase, passing around completely empty buckets.

The foregoing example is non-ideal in the sense that equal areas for the high frequency bunches is not an initial condition well matched to contours in the composite bucket. However, because the span of the initial distribution is only $\pm \frac{3 \pi}{7}$, the mismatch is not extreme. In the immediately following section we consider what can be done when it is desired to coalesce a larger number of equal bunches. The observations made above are useful in that more difficult case as well.

\section{Coalescing More Equal Bunches}

Our second example is much closer to the proton bunch coalescing operation used in the Main Ring. In Table 2 are given the parameters for coalescing of eleven $h=1113$ bunches of $0.2 \mathrm{eVs}$ each into an $h=53$ bucket at $150 \mathrm{GeV}$. The example is extreme; if the Main Ring works anywhere close to its historical 


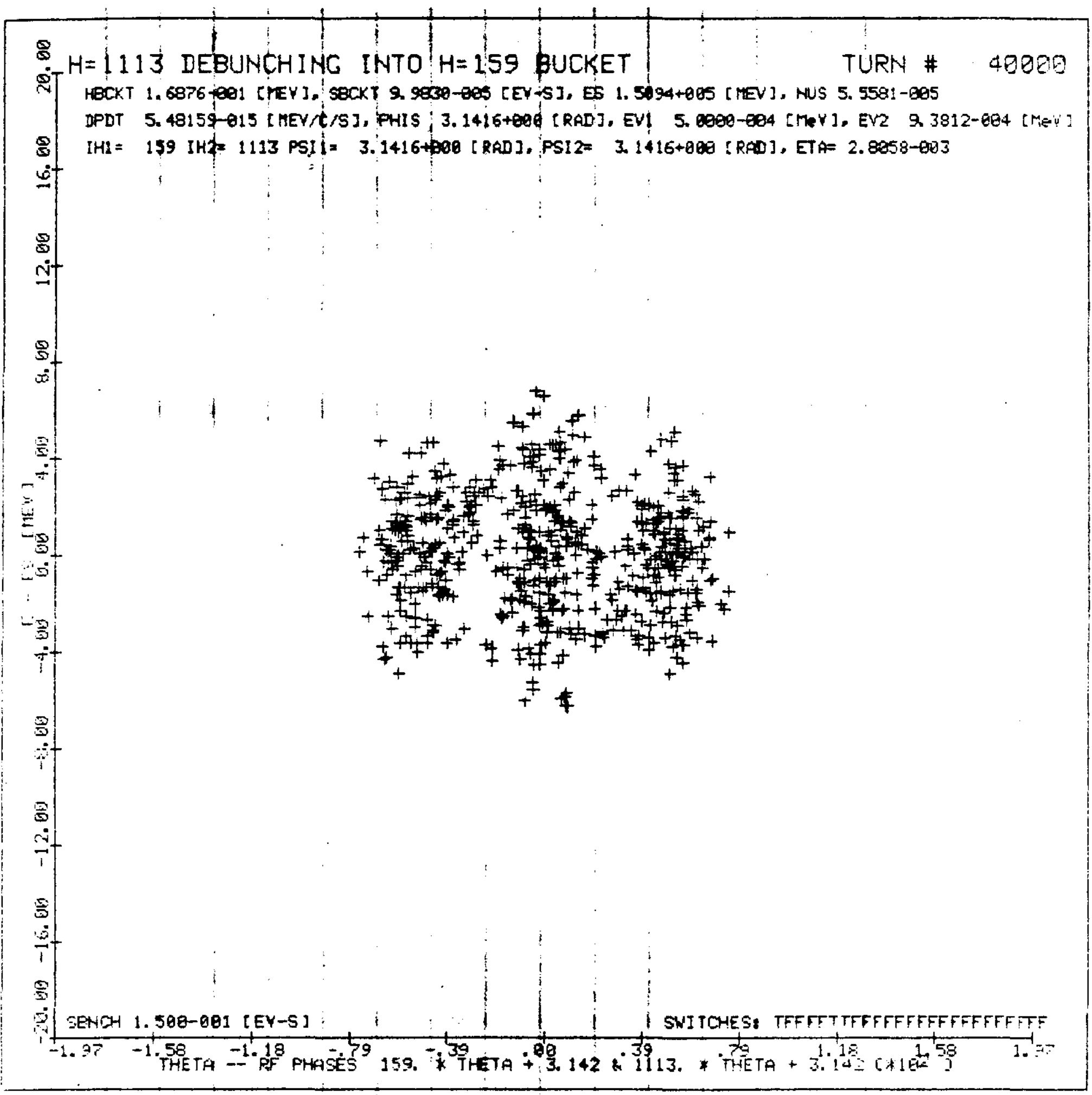

Figure 3: Coalescing from $h=1113$ to $h=159$ at intermediate stage $\left(4 \times 10^{4}\right.$ turns) with nominal $h=159$ voltage 


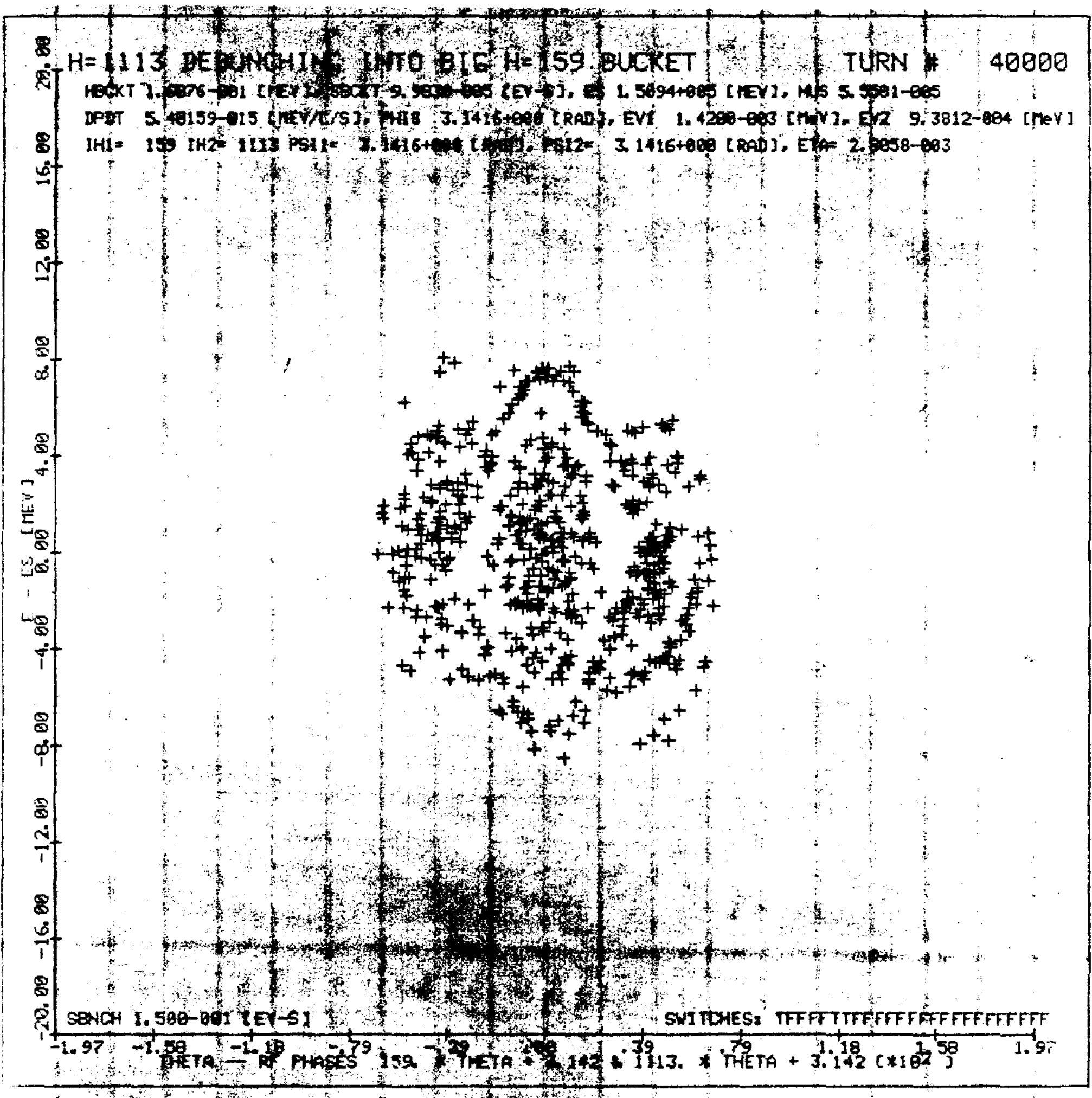

Figure 4: $h=1113$ to $h=159$ at $4 \times 10^{4}$ turns with $h=159$ voltage approximately three times nominal 


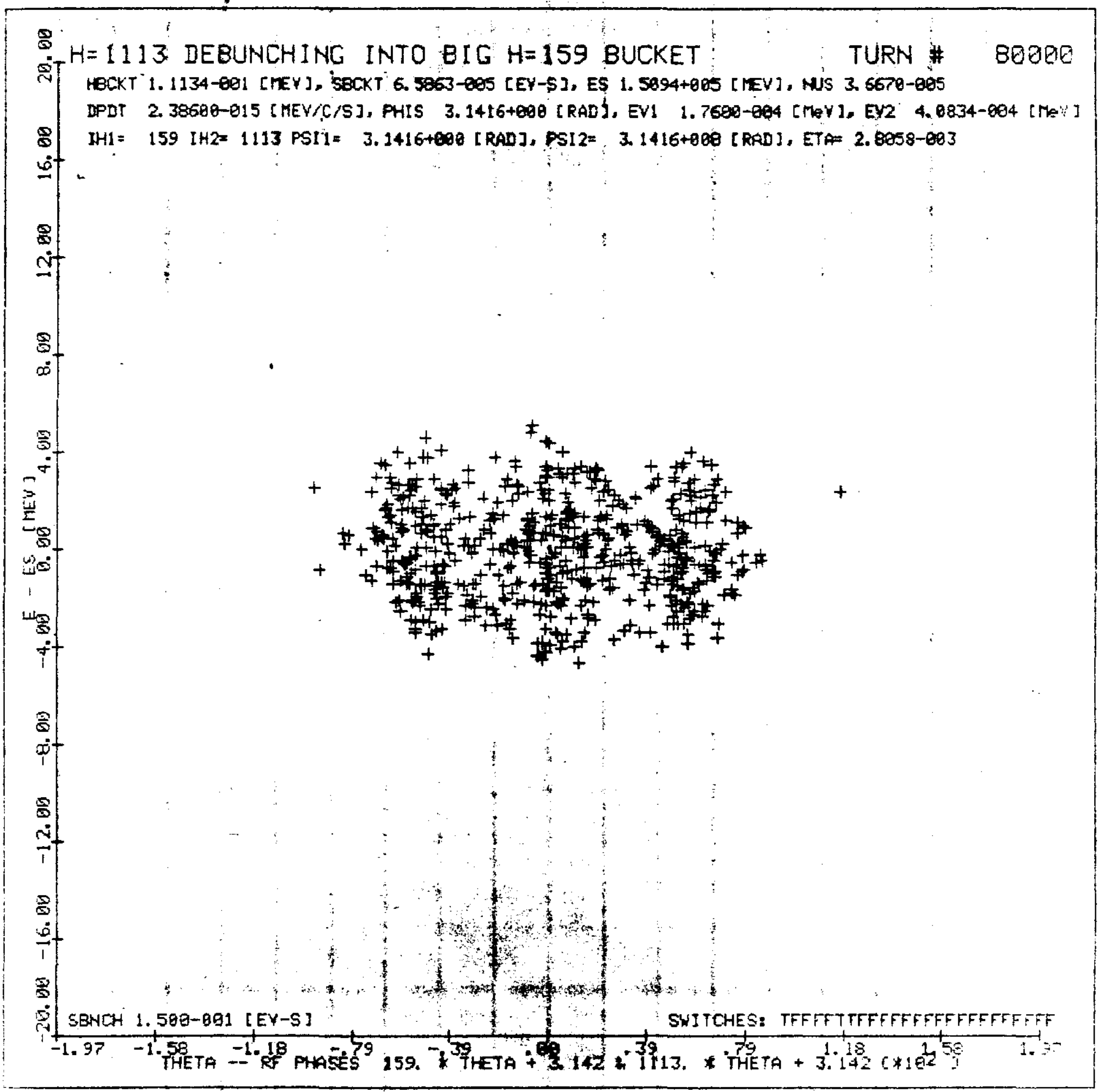

Figure 5: $h=1113$ to $h=159$ at $8 \times 10^{4}$ turns with $h=159$ voltage approximately one-third nominal 
capacity, seven bunches of $0.15 \mathrm{eVs}$ should produce a coalesced bunch of $10^{11}$ protons.

Because the initial distribution is qualitatively rectangular overall it does not seem a good candidate to fit into a standard fixed bucket. Experience of many years standing related to round pegs and square holes leads us to look for a square bucket to contain our coalesced distribution. Happily the $h=106$ system already used to linearize the bunch rotation can be used as well to "square up" the $\mathrm{h}=\mathbf{5 3}$ bucket. The ideal bucket, $i$. e. one producing nearly rectangular contours, would have no rf focusing within the $\pm \frac{11 \pi}{21}$ occupied by the initial bunches. This condition is met precisely at zero phase and approximately over the entire range by an $h=106$ amplitude one half that of the $h=53$ and 180 deg out of phase at the center of the distribution. The complete parameter set is given in Table 2 . The amplitude for $h=53$ has been chosen by exactly the same rule used for choosing the low-frequency amplitude in the previous example, with no adjustment for the second harmonic voltage. Figure 6 is the result of an ESME tracking for $6 \times 10^{4}$ turns. The calculation was carried out to $10^{5}$ turns with iso-adiabatic voltage reduction. The fact that the final $4 \times 10^{4}$ turns did not result in noticeable reduction of the envelope of the distribution implies that the process as specified is not ideally matched throughout. The fact that the coalesced distribution has very nearly the bunch area of the initial distribution implies that the non-ideality has no prctical importance. Figure 7 shows that even eleven fat proton bunches can be caught in a single $h=1113$ bucket after a $\frac{\pi}{2}$ rotation; this would seem to encourage hope that what is actually needed for Tevatron colliding beams is attainable.

\section{Conclusion}

Two examples of the coalescing of equal area bunches into a single bucket at lower harmonic number have been worked out to show that a rather simple rule determines good values for the appropriate of system parameters. Application of this approach in the Fermilab Main Ring requires consideration of further matters like the practicality of controling the of amplitudes and phases and the stability of the resulting low momentum spread distributions; these important matters need more develpment work and more experimental tests. Calculations like those described in this note show that in principle such coalescing processes can proceed with no significant dilution of the longitudinal phasespace area; the importance of beam brightness for colliding beam physics in the Tevatron warrants strong effort to approach this ideal in practice. 


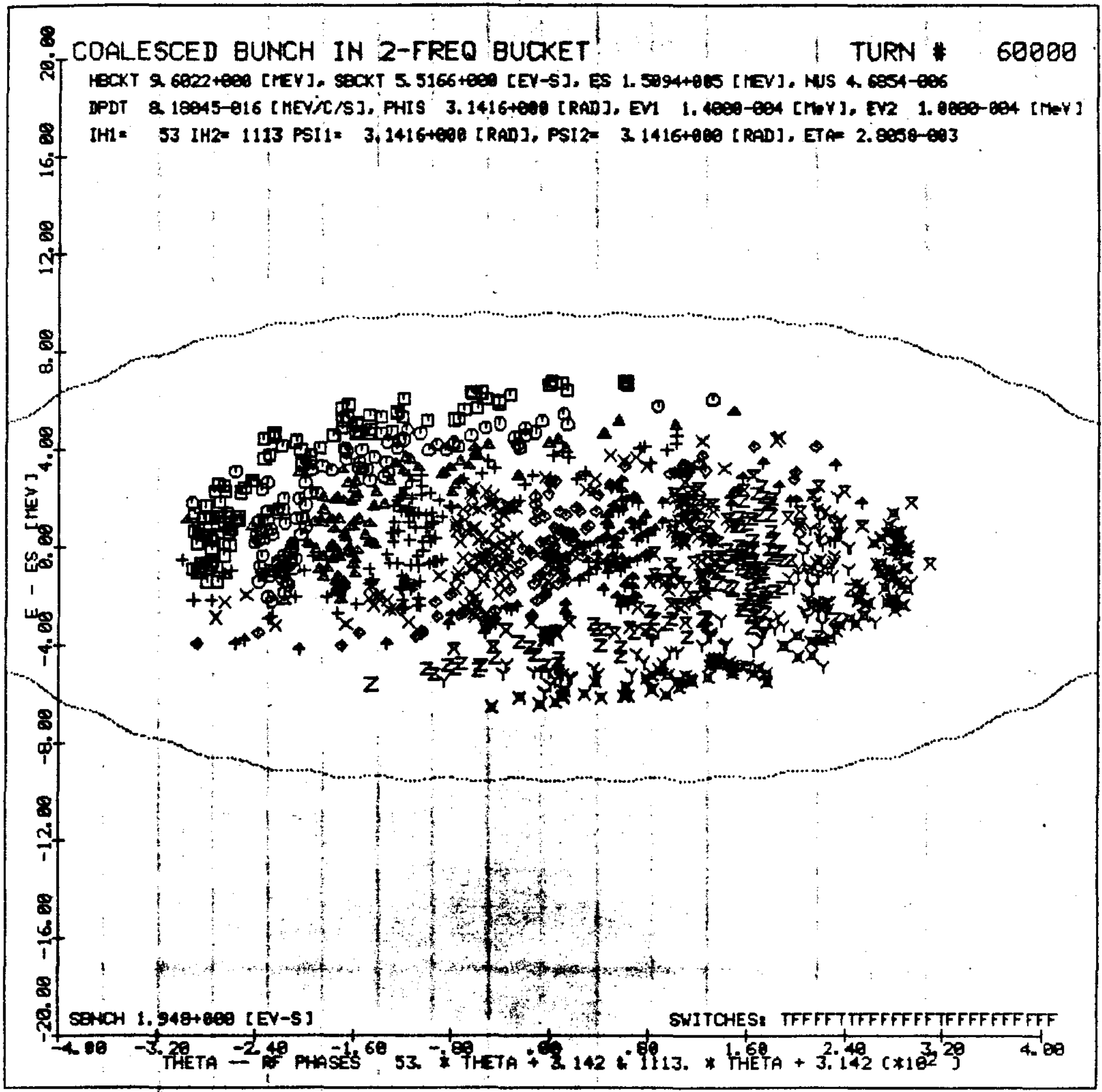

Figure 6: $h=53$ bunch coalesced from eleven $h=1113$ bunches and bucket contour produced by $h=53, h=106$, and $h=1113$ simultaneously 


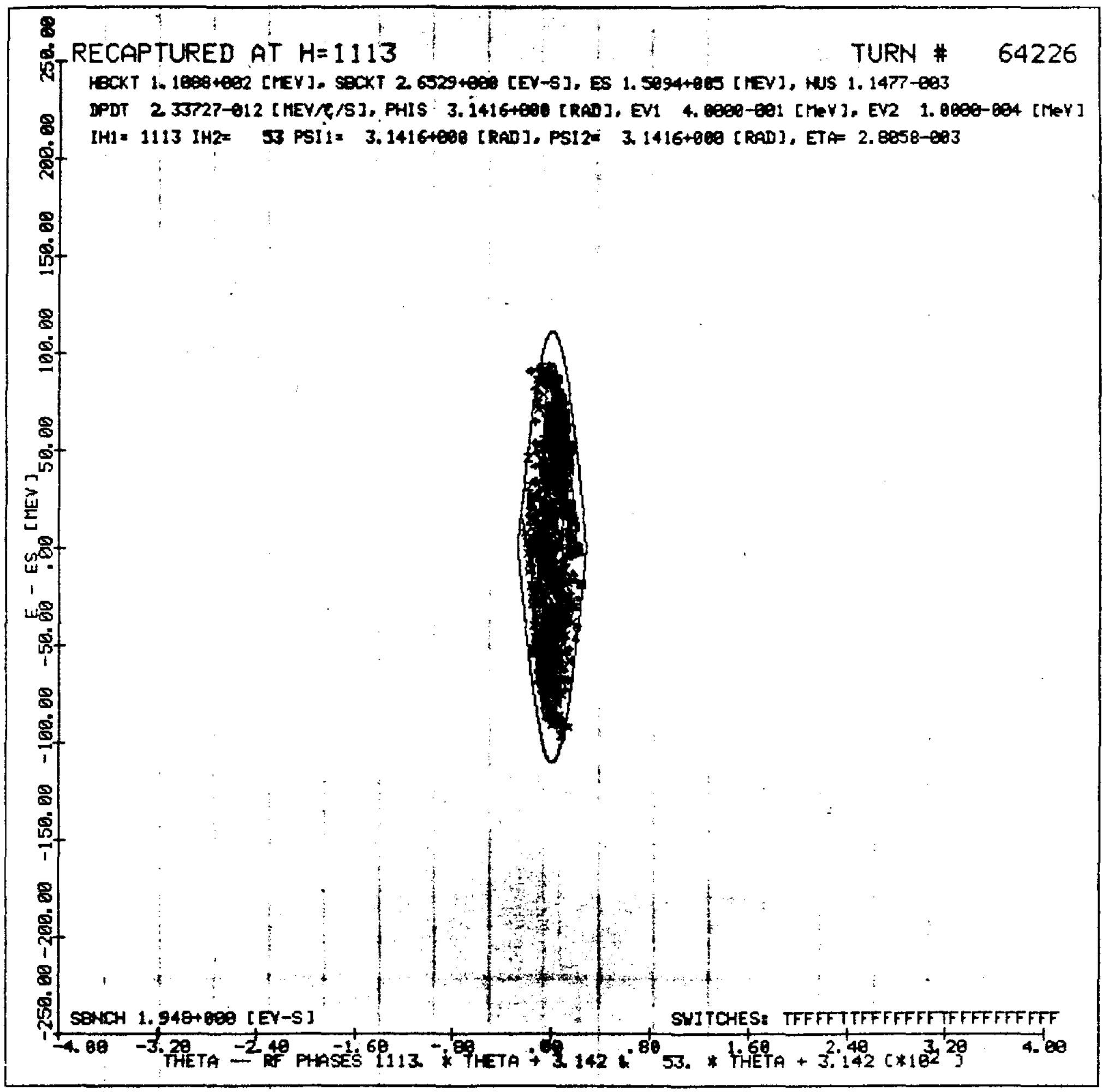

Figure 7: Coalesced bunch after $\frac{\pi}{2}$ rotation and recapture at $h=1113$

11 


\begin{tabular}{|lcr|} 
Orbit circumference $(2 \pi \bar{R})$ & \multicolumn{2}{c}{$6.283 \mathrm{~km}$} \\
Transition gamma $(\gamma T)$ & 18.75 & \\
Harmonic number, $53 \mathrm{MHz}$ system & 1113 & \\
Initial $53 \mathrm{MHz}$ voltage $\left(V_{i}^{(1113)}\right)$ & $10 . \quad \mathrm{kV}$ \\
Initial h $=1113$ bucket area & $0.42 \mathrm{eVs}$ \\
Final $53 \mathrm{MHz}$ voltage $\left(V_{f}^{(1113)}\right)$ & $100 . \quad \mathrm{V}$ \\
Final h=1113 bucket area & $0.06 \mathrm{eVs}$ \\
Number of turns & $6 \times 10^{4}$ & \\
Harmonic number, $2.5 \mathrm{MHz}$ system & 53 & \\
Voltage, $2.5 \mathrm{MHz}$ system $\left(V^{(63)}\right)$ & 140. & $\mathrm{~V}$ \\
Bucket area for h $=53$ & $5.52 \mathrm{eVs}$ \\
Harmonic number, $5.0 \mathrm{MHz}$ system & 106 & \\
Voltage, $5.0 \mathrm{MHz}$ system $\left(V^{(106)}\right)$ & 70. & $\mathrm{~V}$ \\
Number of $h=1113$ bunches & 11 & \\
Initial h $=1113$ bunch areas & $0.2 \quad \mathrm{eVs}$ \\
Final h $=53$ bunch area & $\sim 2.2 \mathrm{eVs}$ \\
& & \\
\hline
\end{tabular}

Table 2: RF System Parameters for $h=1113$ to $h=53$ Coalescing

\section{References}

[1] Design Report Tevatron 1 Project, Fermilab(9/84)

[2] J. A. MacLachlan, Fermilab TM-1274, unpub. 